\title{
Innovación docente y su aplicación al EEES: Emprendimiento, TIC, y Universidad
}

\author{
Gloria JiMÉNEZ MARÍN \\ Universidad de Sevilla \\ gloria_jimenez@us.es \\ Rodrigo ELÍAS ZAMBRANO \\ Universidad de Sevilla \\ rodrigoelias@us.es \\ Carmen Silva Robles \\ Universidad de Cádiz \\ carmensilva.robles@uca.es
}

\begin{abstract}
Resumen:
El objetivo de esta investigación es estudiar el desarrollo de la formación universitaria mediante el emprendimiento, su consideración y puesta en práctica en casos reales con alumnos de la Facultad de Comunicación en Universidad de Sevilla y de la Facultad de Ciencias Sociales y de la Comunicación en Universidad de Cádiz. La investigación, bajo un estudio de casos, se centró en observar las variaciones de la docencia en función de si una asignatura instauraba como metodología docente un proyecto de emprendimiento o no. De este modo, los resultados mostraron un patrón común independientemente de la modalidad formativa de la asignatura y un interés del profesorado en alternar diferentes metodologías.
\end{abstract}

Palabras clave: Emprendimiento; Metodologías docentes; Docencia universitaria; OTRI; Comunicación.

\section{Teaching innovation and its application to the EEES: Entrepreneurship, ICT, and University}

\begin{abstract}
:
The objective of this research is to study the development of university courses through entrepreneurship, consideration and implementation in real cases with students from the Faculty of Communication at the University of Seville and the Faculty of Social Sciences and Communication at University Cadiz. The research, with a case study, focused on observing teaching variations depending on whether a course teaching methodology was as a proposed venture or not. Thus, the results showed a common pattern regardless of the type of training of the subject and teacher interest in alternate different methodologies.
\end{abstract}

Key Words: Communication; Entrepreneurship; OTRI; Teaching methodology; University teaching.

\section{Referencia normalizada:}

Jiménez Marín, G., Elías Zambrano, R. y Silva Robles, C. (2014): Innovación docente y su aplicación al EEES: Emprendimiento, TIC y Universidad. Historia y Comunicación Social. Vol. 19. Núm. Especial Marzo. Págs. 187-196.

Sumario: 1. Introducción, 2. Metodología, 3. Resultados, 4. Conclusiones, 5. Bibliografía 


\section{Introducción}

El emprendedor es una persona con una serie de actitudes, aptitudes y habilidades personales y grupales que le llevan a buscar sus propias salidas a nivel laboral, a nivel familiar, a nivel individual, etc. Porque el emprendimiento es una capacidad del ser humano para salir adelante, y crecer de una manera creativa y con ideas y fórmulas diferentes: es la capacidad de una persona (o grupo de personas) de superar baches o remontar situaciones de formas distintas, en este caso, a nivel empresarial.

El emprendimiento no es un concepto, en absoluto, novedoso: siempre ha estado presente a lo largo de la historia de la humanidad, pero quizás ahora se hace más patente su significado (y necesario): y es que el resurgimiento y adaptación de las diferentes situaciones económicas y cómo éstas han sido superadas con nuevas empresas, nuevas fórmulas que, de una u otra manera, están ayudando a superar las crisis financieras actuales (y pasadas).

La palabra 'emprendimiento' proviene del francés 'entrepreneur', y significa pionero: la capacidad de una persona de realizar un esfuerzo para alcanzar una meta, también usado el término para referirse a una persona que inicia una nueva empresa o proyecto, así como a personas que agregan valor a un producto ya existente.

Partiendo de la acepción que trata al emprendedor como un ser con inquietudes por aprender y mejorar, la que se refiere a la aptitud y actitud de la misma, lo que le permite emprender nuevos retos y nuevos proyectos y, basándonos en la realización de un DAFO, podemos afirmar que un emprendedor es capaz de aprovechar las situaciones de insatisfacción personal para dar la vuelta a esa situación y conseguir, con ello, una situación de oportunidad. Y, en este sentido, es importante destacar el papel de la universidad, que juega un papel fundamental a la hora de dar a sus alumnos una formación específica para que estos estén capacitados para alcanzar sus objetivos.

Los emprendedores, pues, son personas generadoras de ideas que son capaces de desarrollar las competencias necesarias para ser mejores empresarios, mejores personas y mejores generadores de ideas y acciones.

Así, y en este sentido, la formación a nivel universitaria tiene como misión configurar la personalidad y conocimientos del individuo de manera profusa y con calidad y criterio, es decir, enseñarle una profesión además de hacerle pensar, crecer como persona, como adulto formado y con criterio: pensar, en definitiva. Por ello, es interesante destacar que debe plantearse la educación superior con una visión del proceso de enseñanza-aprendizaje, además de como una condición del docente para hacer crecer al alumno que tiene delante. Por esta razón, la educación se encuentra casi en el compromiso de proporcionar un correcto ya adecuado desarrollo de habilidades personales: capacidad de innovar, encuentro de soluciones creativas, o adaptación a entornos cambiantes o, sencillamente, monotonía en el panorama laboral (o incluso personal). 
Pero en los últimos años el planteamiento educativo está desarrollándose en otro sentido: autores como Robinson $(2009,2011)$ o Gerver (2009) afirman y favorecen teorías que procuran una revolución desde su planteamiento inicial: no solo se debería cambiar la organización de los estudios, sino también su finalidad así como el tiempo de dedicación, que debería pasar de ser una formación puntual y con tiempos preestablecidos, a ser una formación durante toda la vida. Toda esta realidad viene recogida en la configuración de los actuales planes de estudios tal y como los consideran los planes de Bolonia (EEES).

Un docente universitario es, pues, un ciudadano de la sociedad del conocimiento que asume el desafío de responder ante los cambios de un modo positivo, con ideas renovadas (o reformuladas -aunque antiguas-) y perspectivas diferentes a la hora de hacer las cosas. Así, con iniciativa y decisión ante las distintas circunstancias, nos atrevemos a afirmar que es la figura del docente emprendedor la que ha desarrollado unas capacidades para el desempeño ciudadano, que es competente, que sabe y sabe hacer, permitiendo, de este modo, un aprendizaje tipo 'Lifelong learnig ${ }^{l}$ '. Esto es: primero hay que desarrollarse como persona para, posteriormente, poder adquirir las competencias humanas, profesionales o específicas para, de este modo, poderlas transmitir.

En este sentido, el desarrollo de estas aptitudes debe encontrarse en un contexto universitario para que el entorno sea favorable y los estudiantes se desenvuelvan en el paso de adolescentes a adultos, con un aprendizaje activo y significativo porque, en definitiva, es lo que permitirá el uso de las competencias en otros espacios y momentos $\mathrm{y}$, por tanto, de la forma en que podrán transmitir y trasladar los conocimientos y habilidades aprendidas y aprehendidas.

La calidad de la educación universitaria debe estar en concordancia con la formación que se les brinda a los futuros egresados. Por y para ello, el docente debe reflexionar sobre su quehacer pedagógico y metodológico, sobre la pertinencia y aplicabilidad de lo que está enseñando. De este modo, el enfoque que se le viene otorgando a este nuevo paradigma de educación superior está orientado a la formación basada en competencias. Así, tal y como expresan Sáenz, Zambrano et al. (2008):

La formación basada en competencias constituye una propuesta que parte del aprendizaje significativo y se orienta a la formación humana integral como condición esencial de todo proyecto pedagógico; integra la teoría con la práctica en las diversas actividades; promueve la continuidad entre todos los niveles educativos y entre estos y los procesos laborales y de convivencia; fomenta la construcción del aprendizaje autónomo, orienta la formación y el afianzamiento del proyecto ético de vida; busca el desarrollo del espíritu emprendedor como base del crecimiento personal y del desarrollo socioeconómico y fundamenta la organización curricular con base en proyectos y problemas, trascendiendo de esta manera el currículo basado en asignaturas compartimentadas.

1 'Lifelong learning': término de origen anglosajón para designar la Educación Permanente, un término y movimiento que pretende llevar la educación a todos los niveles y estados del ser humano (no necesariamente enseñanza de adultos, sino enseñanza a cualquier nivel). 
Por ello, y siguiendo esta reflexión, es fácil afirmar que una formación de este tipo demanda del docente un papel diferente al tradicional, al del profesor magister, que imparte clases magistrales: porque ahora el centro del proceso no es el profesor, sino el estudiante, cumpliendo el docente una función de coordinador, acompañante, guía, asesor y orientador, más que de profesor en un sentido literal. El verdadero protagonista el estudiante.

Hay, pues, un cambio radical en su quehacer pedagógico, en la todavía imperante mentalidad, en el sistema de evaluación, etc. Y para conseguir esta formación el docente debe generar esferas en sus clases que propicien critica, trabajo en equipo, autoformación y, por supuesto, reflexión.

Así, una buena estrategia para obtener autoformación por parte de los estudiantes (siempre tutorizada por el profesor) es el trabajo independiente que venga bien definido en los diferentes diseños curriculares con créditos académicos y extra académicos (SET: Suplemento Europeo al Título). Por todo ello, debemos orientar el trabajo del alumno fomentando la formación de competencias en los estudiantes. ¿Cómo?

Guiando al estudiante en la consecución de recursos para la realización de actividades sugeridas por parte del docente.

Orientando a los estudiantes para que construyan las estrategias de cada uno de los saberes de las competencias.

Estableciendo las estrategias pedagógicas con la participación de los mismos estudiantes.

Centrando el aprendizaje en os estudiantes y no en la enseñanza ni en el profesor.

Acordando con los estudiantes las competencias a desarrollar teniendo en cuenta las expectativas y requerimientos socio-ambientales y laborales

\subsection{Objetivos}

Partiendo de la base de que el alumno es el epicentro del proceso didáctico (y dejando de lado su papel de sujeto pasivo en el aula para estar mucho más implicado en el proceso de aprendizaje), también podemos afirmar que el estudiante es el responsable de sus propias relaciones de ayuda con el profesor y con el mundo exterior que le rodea. Por esta razón, nuestro objetivo busca varios propósitos:

En primer lugar una búsqueda de autoempleo, así como una mejora del cv personal del alumnado a punto de egresar de los estudios universitarios. También orientar a los alumnos para su solvencia a la hora de afrontar el problema de la búsqueda de empleo, así como crear una posible idea de negocio rentable, con lo que, al mismo tiempo, perseguimos otros empeños:

- Acercamiento a la realidad empresarial.

- Búsqueda de la creatividad 
- Trabajo en equipo

- Empatía con el entorno

- Capacidad de adaptación

- Iniciación a la participación de concursos públicos y privados

- Identificar las aportaciones que introduce el "Aprendizaje basado en Proyectos" en la formación de titulados universitarios.

- Identificar estrategias concretas que puedan mejorar el proceso de enseñanza-aprendizaje e incorporarla al concepto de créditos ECTS.

- Inculcar a los alumnos el sentido de responsabilidad y cooperación en un escenario de realidad.

Lagardera (1992, p.55) afirma que "nada educa per se y, al mismo tiempo, todo en la vida es susceptible de promover una situación educativa". También Duart (2002) afirma que:

la educación no es una acción neutra. Los valores éticos se encuentran en la razón y el objetivo de la acción educativa. Aprender es ante todo educarse, formar el propio ser. Y este es un proceso que se desarrolla de forma permanente a lo largo de nuestras vidas.

Por ello, desde la concepción de esta investigación estudiamos la educación de una forma distinta y los principios del emprendimiento los aplicamos a los estudios de Publicidad y RR.PP. y de Comunicación Audiovisual. Porque encontramos en esta forma de trabajo una opción distinta a los actuales modelos de la práctica docente, por otra parte, cada vez más en boga, circunscrito a la situación económica y social en la que nos encontramos.

\section{Metodología}

Mediante el trabajo empírico tratamos de determinar los factores más relevantes desde el punto de vista de los distintos colectivos que conforman el contexto universitario. Por tanto, este análisis se ha realizado desde una doble perspectiva: por una parte, desde el punto de vista de la demanda, personas afectadas que tienen interés en el tema, y por otra, estudiando la oferta actual de información y ayudas a las iniciativas de creación de empresas, oficinas de transferencia de resultados de investigación (OTRI) o iniciativas de concursos empresariales.

Así, distinguimos dos fases principales en este estudio: En primer lugar, la relativa a la recogida directa de información, efectuándose entrevistas en profundidad a estudiantes, emprendedores universitarios y profesores universitarios. 
La muestra de estudiantes está compuesta por estudiantes del campus de Cartuja en Universidad de Sevilla y de Campus de Jerez de la Frontera, en la Universidad de Cádiz. A su vez, estas muestras se subdividieron en estudiantes motivados (es decir con alguna idea previa de emprender un negocio) y no motivados, entrevistándose a un total de veintiséis estudiantes: trece motivados y trece no motivados.

Respecto a los emprendedores, estos fueron alumnos egresados de las titulaciones de Publicidad y RR.PP. o Comunicación Audiovisual de alguna de las dos universidades. Asimismo, entrevistamos a diversos profesores universitarios con la característica de tener algún tipo de vinculación, bien empresarial, bien con el emprendimiento, suponiendo un total de 6 profesores universitarios.

Una vez realizadas, grabadas y transcritas las entrevistas, procedimos a la recogida sistemática de información de las principales instituciones que tienen programas de incentivación y promoción de emprendedores o bien se limitan a asesorar y dar información, tanto en Sevilla como en Cádiz. La recogida de esta información se seleccionó según su interés para los estudiantes universitarios.

Para analizar la información obtenida, y dado que utilizamos una técnica cualitativa como es la entrevista en profundidad, se utilizó el análisis de contenido. De este modo, las entrevistas transcritas fueron analizadas y agrupadas según bloques de información, estructurando las respuestas según los comentarios de los entrevistados, que compusieron el modelo final propuesto en el apartado de resultados.

Asimismo, se utilizaron las propias verbalizaciones de los sujetos como dato cualitativo para apoyar la idea resaltada en los resultados.

\section{Resultados}

Los resultados obtenidos los analizamos en base a grandes bloques temáticos, por los diferentes temas tratados:

- Factores que pueden incitar u obstaculizar la creación de una empresa propia;

- Factores que definen a los individuos emprendedores y a los no emprendedores.

- Papel de la Universidad en el proceso de aprendizaje para los emprendedores.

Los principales resultados obtenidos fueron los siguientes: En relación a los estudiantes universitarios, y una vez entrevistados, partimos de la gran diferencia existente entre estudiantes a nivel de información sobre emprendimiento, ayudas, etc. Y partimos del hecho de que los estudiantes, por sí mismos, en general tienen poca actitud emprendedora (esto se acrecienta en carreras más humanísticas). En cuanto a los factores que ayudan a emprender, observamos que muchos son los factores que pueden favorecer la intención del estudiante de crear su propio negocio. Así, entre los 
factores de sociocultural, se encuentran la búsqueda de trabajo o la tradición familiar. Sin embargo, entre los factores de carácter psicológico destacan:

- Posesión del 'espíritu emprendedor': Los estudiantes que se ven a sí mismos como emprendedores comentan que es algo que se ve reflejado en las tareas y el funcionamiento diario, en la manera de organizar y enfocar lo que te rodea. Del mismo modo, los no emprendedores parecen atribuirlo a un carácter, una manera de ser.

- Deseo de autorrealización: los propios estudiantes consideran que los sujetos que realmente tienen o poseen un proyecto y se vinculan a él como proyecto personal de desarrollo, son los que se motivarán para ser emprendedores.

- Posible aparición de una idea.

- Sensación de independencia y ser su propio jefe.

También tienen importancia otros factores, en este caso, negativos, como son los factores que suponen una barrera para el emprendimiento. Así, los factores que impiden a emprender son de varios tipos:

Tabla 1

\begin{tabular}{|l|l|}
\multicolumn{1}{|c}{ Psicológicos } & \multicolumn{1}{c|}{$\begin{array}{c}\text { Derivados del entorno sociocultural y las } \\
\text { instituciones }\end{array}$} \\
\hline $\begin{array}{l}\text { Miedo a la asunción de riesgos } \\
\text { (económicos, personales, sociales...). }\end{array}$ & $\begin{array}{l}\text { Problemas económicos, posibilidad } \\
\text { de obtención de la financiación inicial } \\
\text { necesaria. }\end{array}$ \\
\hline $\begin{array}{l}\text { Auto percepción de inmadurez, falta de } \\
\text { cualificación y experiencia. }\end{array}$ & Excesiva burocracia. \\
\hline
\end{tabular}

El perfil del emprendedor, frente al del no emprendedor, se dibuja de una manera clara y diáfana. Se hace bastante énfasis en los aspectos psicológicos acerca del perfil personal tanto del emprendedor como del no emprendedor. Por un lado, la personalidad creativa e independiente, así como el espíritu empresarial de los individuos, potencia ese perfil de emprendedor. Desde un punto de vista social, se perciben tres elementos importantes respecto al rol que desempeña el emprendedor en la sociedad actual como son el avance social, la riqueza generada o el sacrificio social.

Por último, desde una perspectiva gerencial, se hace alusión a la formación o experiencia que posee el emprendedor. Se aprecia como característica del mismo su capacidad para hacer frente a situaciones desestructuradas e, incluso, se aprecia una preferencia por el dinamismo y los cambios para alejarse de la monotonía. En los estudiantes motivados se observa esta tendencia y, su cultivo durante su estancia en la universidad facilitará su futura tarea emprendedora.

Pero, por otra parte, también analizamos y observamos el perfil del no emprendedor. $\mathrm{Y}$ es que, los estudiantes apuntaron fundamentalmente factores psicológicos frente a otro tipo de factores. Así, los estudiantes apuntaron que el estudiante no 
emprendedor se asocia a ciertos rasgos de personalidad o elementos innatos, estáticos y pasivos, basándose en una posible falta de creatividad, entre otras características.

También recogimos las opiniones de los estudiantes acerca de los proyectos empresariales propuestos por el profesorado, en distintas asignaturas, sobre emprendimiento y creación de empresas. En este sentido, destacamos que los estudiantes motivados muestran claramente una mayor información que los no motivados. A nivel psicológico, el único factor encontrado es el rol de la Universidad como fuente motivadora a la hora de impartir clases, charlas informativas o sugiriendo proyectos empresariales como método de evaluación. A nivel sociocultural, se destaca la falta de modelos a seguir, coherentes y responsables, así como éticos. El alumnado plantea más sesiones informativas y, sobre todo, formación específica sobre la creación de una empresa como proyecto real. También sugirió el alumnado la apertura de un servicio de atención al estudiante, donde se pueden potenciar las iniciativas emprendedoras que demanden los estudiantes universitarios.

Se realizaron también entrevistas a emprendedores, de donde se extrajeron los facotres que ayudan a emprender. Así, los emprendedores entrevistados hicieron hincapié en la idea de negocio como motor fundamental. Desde un punto de vista psicológico, los emprendedores a los que se les consultó comentan como principal elemento los conocimientos y experiencia laboral previos a la creación del negocio y puesta en marcha. Sin embargo, es curioso que también existe un cierto número de emprendedores que, careciendo de experiencia laboral, plantean la posibilidad de emprendimiento debido a motivaciones internas, innatas.

Desde un enfoque sociocultural los emprendedores destacan la situación laboral actual española y los modelos y valores familiares como elementos que pueden favorecer la aparición de nuevos emprendedores.

Por el contrario, las barreras encontradas por los posibles emprendedores son, básicamente, de carácter institucional: y es que los emprendedores se quejan de los problemas iniciales que encuentran a la hora de desarrollar su idea, a nivel financiero, carencia de fuentes de información fiables o burocracia rebuscada a la hora del montaje de la estructura empresarial (con la consecuente pérdida de tiempo y esfuerzos).

\section{Conclusiones}

Cuando se les pidió a los propios emprendedores que definiesen cuáles de sus características principales les describían y distinguían de individuos no emprendedores, plantearon fundamentalmente caracteres psicológicos: implicación con los proyectos, actitudes emprendedoras, creatividad, ambición, capacidad laboral, capacidad de toma de decisiones, autonomía, etc. 
Por su parte, entre las características tipo de los no emprendedores, los términos más utilizados fueron: "estereotípico funcionario", "conformismo", "baja tasa de rendimiento", "pasividad", etc.

A nivel psicológico, algunos de los comentarios más repetidos fueron: "la Universidad debe fomentar más jornadas y actividades o seminarios" o incluso "que desde la universidad se oriente al alumno".

A nivel institucional los entrevistados comentaron el papel de la universidad desde un punto de vista positivo, a través de las diversas fundaciones Universidad-Empresa como facilitador de contacto con otras instituciones. Sin embargo, también tuvieron críticas contra la universidad al referirse a ella como "a veces es excesivamente teórica, hace falta más mundo real".

Entre los factores psicológicos que incitan a ser emprendedor, según el punto de vista de los profesores universitarios entrevistados, destaca la existencia de una motivación interna, una necesidad personal, una inquietud o herencia familiar. Otros elementos que motivan fueron de carácter más sociocultural que de otro tipo, como la necesidad laboral-económica, la existencia de un entorno familiar propicio o los contactos empresariales existentes que fomenten la creación de una idea de negocio.

Como obstáculo o barreras para emprender, los profesores universitarios señalan la politización de las instituciones, el perfil pasivo del no-emprendedor o incluso la incertidumbre y miedo al riesgo. Respecto a qué aspectos distinguen a un individuo Emprendedor del no emprendedor, desde el punto de vista psicológico se señalan el entusiasmo (frente a la pasividad), la ilusión (frente a la pusilanimidad), el espíritu emprendedor (frente al espíritu no emprendedor) o la capacidad de observación (frente a la incapacidad de observación).

Por todo ello, y como resultado y conclusión a este estudio, fruto del II Plan Propio de Docencia de la Universidad de Sevilla y en colaboración con una convocatoria de Proyectos de Innovación Docente de la Universidad de Cádiz, nos encontramos inmersos en la creación de una oficia de atención al emprendedor en el Campus Cartuja de la Universidad de Sevilla y en el Campus de Jerez de la Universidad de Cádiz. Este proyecto, en pleno desarrollo ha contado con financiación específica para apoyar al emprendedor mediante la organización de jornadas y seminarios, la creación de un premio a la mejor idea empresarial, la aportación de información burocrática para la puesta en marcha de la empresa o la tutorización de proyectos empresariales.

Apoyar a un emprendedor supone crear riqueza económica y social y un trabajo de incertidumbre en relación al entorno cambiante en el que nos movemos (aunque no por ello peligroso). En este sentido, los investigadores de este proyecto, creemos firmemente en el poder del espíritu empresarial y la autorrealización como fuerzas generadoras de empleo y de bienestar social y de la comunidad. 


\section{Bibliografía}

ALDRICH, H. \& RUEF, M. (2006). Organizations evolving (2nd ed.). London: Sage. CEA, M.A. (2001). Metodología cuantitativa. Estrategias y técnicas de investigación social. Madrid: Síntesis sociología.

DUART, J.M. (2000). Aprender en la virtualidad en AA. VV. Aprender. Barcelona: Gedisa Editorial.

GERVER, R. (2010). Creando hoy las escuelas del mañana: la educación y el futuro de nuestros hijos. Madrid: SM.

KRIPPENDORF, K. (1990). Metodología de análisis de contenido. Teoría y práctica. Barcelona: Paidós Comunicación.

LUNDWALL, B. (2002): The University in the Learning Economy. En DRUID, 2. Disponible en http://www.druid.dk/wp/pdf_files/02-06.pdf. [Consultado el 15 de septiembre de 2013].

OLSEN, H. (2004). The Resurgence of Social Entrepreneurship. Fraser Forum, 21-22

ROBINSON, K. (2011). Redes. El blog de Eduard Punset. Disponible en: http:// www.redesparalaciencia.com/wp-content/uploads/2011/03/entrev87.pdf. [Consultado el 26 de agosto de 2013].

ROBINSON, K. (2009). The Element. Barcelona: Grijalbo.

ROBINSON, K. (2001). Fuera de nuestras mentes: Aprender a ser creativo. Capstone Editorial.

SALINAS, J. (1995): Organización escolar y redes: Los nuevos escenarios de aprendizaje. En Cabero, J. y Martínez, F. (1995): Nuevos canales de comunicación en la enseñanza. Madrid: Centro de Estudios Ramón Areces, pp. 89-118.

SÁENZ GARZA, Moisés; ZAMBRANO CHÁVEZ, Nancy; TORRES MUÑOZ, Otoniel; PEREYRA LUNA, Marina; HERNÁNDEZ CONTRERAS, Ely (2008): Sistematización de la enseñanza: formación basada en competencias, en Objetos de aprendizaje - Escuela Superior de Graduados. Disponible en: http:// www.slideshare.net/guest7be $72 \mathrm{~d} /$ formacin-basada-en-competencias-447490. [Consultada el 28 de agosto de 2013].

SALINAS, J. (1997): Nuevos ambientes de aprendizaje para una sociedad de la información. En Revista Pensamiento Educativo, $\mathrm{n}^{\mathrm{o}} 20$. Pontificia Universidad Católica de Chile, pp. 81-104. 